\title{
Effects of CT images with different reconstruction energy on radiotherapy planning for patients with non-small cell lung cancer
}

\section{Tian Xiufang}

Shandong Qianfoshan Hospital https://orcid.org/0000-0003-3468-0246

Liu Kun

Shandong Qianfoshan Hospital

Wang Jing

Hubei University of Medicine

Zhang Jiandong

Shandong Qianfoshan Hospital

Yong Hou ( $\nabla$ 15562689543@163.com )

Shandong Qianfoshan Hospital https://orcid.org/0000-0002-7937-3891

Research article

Keywords: Energy spectrum CT, Radiotherapy, Non-small cell Lung Cancer(NSCLC), Dosimetry

Posted Date: August 24th, 2020

DOI: https://doi.org/10.21203/rs.3.rs-51290/v1

License: (c) (1) This work is licensed under a Creative Commons Attribution 4.0 International License.

Read Full License 


\section{Abstract \\ BACKGROUND}

With the development of the CT technology, multi-energy technology is applied to CT imaging, which improves the time resolution, spatial resolution and density resolution of CT system. It enables the CT imaging system to achieve clearer image display under safe and low-dose conditions, so that the disease can be displayed more quickly, and the disease can be identified more early and more clearly.

\section{METHODS}

38 non-small cell lung cancer patients were selected for energy spectral scanning. All energy spectral images obtained were transferred to the DiscoverTM CT post-processing workstation to generate $40 \mathrm{keV}$, $60 \mathrm{keV}, 80 \mathrm{keV}, 100 \mathrm{keV}, 120 \mathrm{keV}$, and $140 \mathrm{keV}$ single-energy images. Then the single-energy images were imported to Eclipse, and the oncologist contours the target area and organs at risk (OARs) on the singleenergy images described above, and then the physicist designed radiotherapy plans to perform statistical analysis on the tissue CT value and target volume of each single-energy image, and to compare dosimetry of different plans about the organs at risk and the target area.

\section{RESULTS}

The CT values of GTV, heart, lung, and spinal cord of different energy CT images are statistically different $(P<0.05)$. Among them, the CT value of each tissue obtained in the $40 \mathrm{keV}$ group is the largest, and the $\mathrm{CT}$ value decreases with the increase of energy. There were no statistically significant differences in the homogeneity index $(\mathrm{HI})$, the conformity index $(\mathrm{Cl})$, the maximum dose, the minimum dose and the average dose of the gross tumor volume (GTV) delineated on CT images of different energy $(P>0.05)$, as well as the organs at risk.

\section{CONCLUSIONS}

When CT images of different energies obtained from energy spectral CT scans are used in the design of radiotherapy planning, there are no significant differences in target area outlines and doses caused by energy factors, but the differences in tissue CT values have statistical significant.

\section{Background}

With the development of CT technology, spectral CT has been widely used in recent years, which include in the security check, calculi ingredient analysis, diagnosis of gout and bone marrow edema, and the early diagnosis of tumors, etc[1-7]. Compared with traditional CT, spectral CT has a variety of advantages, including the ability to generate energy decay curves, iodine-based maps, water-based maps, and virtual 
plain scans, which lays a broad application prospect for qualitative diagnosis and quantitative analysis of lesions. At present, the application of spectral CT is mainly used in chest and abdomen examinations. The advantage of virtual plain scan can not only reduce the patient's exposure dose, and reduce the error caused by the inconsistency of the measurement of the area of interest before and after the scan, but also conduct a differential analysis of the nature of the lesion [8-9]. Energy spectrum CT uses a single Xray tube, spectral imaging through single-source instant $\mathrm{kV}$ switching technology, and determines the attenuation coefficient of the voxel in the energy range of 40-140 keV based on these two energy data, and further obtains 101 single-energy images. This relatively pure single-energy image can greatly reduce the impact of hardening artifacts and obtain a relatively pure CT value image, that is, the CT value is more consistent and reliable regardless of the position, scan in the entire visual field, or patient [10]. In the image processing, different substances are decomposed into matrix pairs based on photoelectric effect, and the electron density, effective atomic number, and matrix distribution density are calculated to provide more diagnostic information for the clinic, which expanded the diagnostic application of CT [11]. The development of spectral CT technology also poses new challenges to radiotherapy simulation positioning technology, that is, how to apply spectral CT technology to improve the accuracy of CT valuetissue parameter conversion, thereby improving the accuracy of radiotherapy.

Before radiotherapy, the patient needs to perform a positioning scan on a CT simulator to obtain CT images containing the patient's anatomy and position information and then imported into the radiotherapy planning system. The treatment planning system obtains the conversion curve of CT value and relative electron density of the tissue from the CT images, and, the inhomogeneous tissue needs to be calibrated based on the relative electron density of the tissue, and then the dose calculation is performed [12-14]. In order to obtain clear CT images, the patient's scanning conditions will be different during simulated positioning scans, such as scan voltage, scan current, or scan layer thickness. These will affect the CT value and the relative electron density conversion curve, which will affect the patient's dose calculation of the radiotherapy plan. This study used single-energy images obtained by spectral CT to study the effects of CT images of different energies on the radiotherapy plan for patients with nonsmall cell lung cancer, to guide the setting of scanning conditions during radiotherapy simulation positioning, and to reduce the influencing factors of dose calculation in radiotherapy plan in order to improve the accuracy of radiotherapy.

\section{Methods}

\subsection{Case presentation}

Eight patients with lung non-small cell lung cancer confirmed by pathological biopsy were selected, including 23 cases with lesions on the left and 15 cases on the right lung. The male to female ratio was 21:17. The age range of the selected patients is $55-75$ years, with an average age of 61.2 years.

\subsection{Equipment and software}


All inspections use the gemstone (DiscoverTM CT 750HD, GE Healthcare, USA) energy spectrum scanning mode. The planning system is the Eclipse 10 radiotherapy planning system.

\subsection{Positioning and scanning}

The patients took a supine position with the head advanced, and the scanning range was from the thorax entrance to the diaphragm level, including the energy spectrum scan of the entire lung. Scanning conditions: The tube voltage is instantaneously switched from $40 \mathrm{kVp}$ to $140 \mathrm{kVp}$, the tube current is $550 \mathrm{~mA}$, the rotation time is $0.8 \mathrm{~S}$, the layer thickness is $5 \mathrm{~mm}$, while the spacing is $5 \mathrm{~mm}$, the reconstruction interval is $1.25 \mathrm{~mm}$, and the pitch is $1.375 \mathrm{~mm}$.

\subsection{Post-processing of CT images}

Transfer all energy spectrum imaging data to the post-processing workstation (AW4.5), and reconstruct to generate single-energy images of $40 \mathrm{keV}, 60 \mathrm{keV}, 80 \mathrm{keV}, 100 \mathrm{keV}, 120 \mathrm{keV}, 140 \mathrm{keV}$, then transfer the single-energy images to the Eclipse 10 treatment planning system .

\subsection{Contouring of target areas and OARs}

According to the ICRU83 report and the RTOG1106 consensus, senior clinical physicians contoured the target areas and organs at risk on the single-energy CT images described above [15]. GTV includes the primary tumor and metastatic lymph nodes in the mediastinum and supraclavicular region determined by the images. The clinical target volume(CTV) is $3 \mathrm{~mm}$ of the upper and lower ends of GTV, $5 \mathrm{~mm}$ of axial extension. Then generate PTV by expansion of $8 \mathrm{~mm}$ in all directions from the CTV. The OARs, such as the lungs, spinal cord, and heart were contoured respectively.

\subsection{Planning techniques}

The plans were generated using the Eclipse10 (Varian Inc) treatment planning system. All of the treatment plans were delivered using a 6-MV photon beam. 7-Field intensity modulated radiation therapy(IMRT) plans were completed by senior physicists, and physicians with deputy directors or above have reviewed and evaluated the uniformity of target dose and organ-endangered doses through some dosimetry-related indicators. All patients had a PTV dose of $60 \mathrm{~Gy} / 30$ fractions and the treatment planning objectives were to cover $95 \%$ of the planning target volume a maximum dose of $120 \%$. The plans of all different energy CT images of the same patient uses the same field energy, field direction, optimization parameters, and dose calculation algorithm (AAA). On a single-energy image, a plan that meets the prescription requirements and endangers the organ limit is used. And based on this plan template, the plans of other energy groups were designed separately. For the OARs, the planning objectives were maximal doses of $45 \mathrm{~Gy}$ for the spinal cord. $\mathrm{V}_{40}<30 \%, \mathrm{~V}_{30}<40 \%, \mathrm{D}_{\text {mean }}<25 \mathrm{~Gy}$, for the heart. $V_{5}<60 \%, V_{20}<30 \%$ and $V_{30}<20 \%$ for both lungs. These planning objectives for the OARs were uniform for all patients and were established following the strictest constraint recommendations to assure the lowest possible dose delivered to normal tissues.

\subsection{Evaluation parameters}


The target area HI refers to ICRU83 report and defines as follows:

$H I=\left(D_{2 \%}-D_{98 \%}\right) / D_{50 \%}(1)$

In which, $D_{2 \%}$ represents the irradiation dose received by $2 \%$ of the target volume, $D_{98 \%}$ represents the irradiation dose received by $98 \%$ of the target volume, and $D_{50 \%}$ represents the irradiation dose received by $50 \%$ of the target volume.

And the $\mathrm{Cl}$ is:

$\mathrm{Cl}=\left(\mathrm{V}_{\mathrm{t}, \mathrm{ref}} / \mathrm{V}_{\mathrm{t}}\right) \times\left(\mathrm{V}_{\mathrm{t}, \mathrm{ref}} / \mathrm{V}_{\mathrm{ref}}\right)(2)$

Where $V_{t}$ is the volume of the target area, $V_{t, \text { ref }}$ is the volume of the target area surrounded by the reference isodose line surface, and $V_{\text {ref }}$ is the volume of all areas surrounded by the reference isodose line. The closer the $\mathrm{Cl}$ value is to 1 , the better the conformal coverage is.

Compare the CT value of tissues and GTV volume with different energy CT images. Evaluate $\mathrm{HI}, \mathrm{Cl}$, maximum dose $D_{\text {max }}$ minimum dose $D_{\text {min }}$, and average dose Dmean in the target area. For the OARs, the planning objectives were maximal doses of $45 \mathrm{~Gy}$ for the spinal cord. Evaluate $\mathrm{V}_{40}, \mathrm{~V}_{30}$, and $\mathrm{D}_{\text {mean }}$ for the heart, and $\mathrm{V}_{5}, \mathrm{~V}_{20}$ and $\mathrm{V}_{30}$ for both lungs.

\subsection{Statistical methods}

The data were divided into 6 groups based on different energies, which are $40 \mathrm{keV}$ group, $60 \mathrm{keV}$ group, $80 \mathrm{keV}$ group, $100 \mathrm{keV}$ group, $120 \mathrm{keV}$ group, and $140 \mathrm{keV}$ group. SPSS 20.0 software was used to analyze the measurement data. All measurement data were firstly tested for normality. When the measurement data obey the normal distribution and the variance is homogeneous, one-way analysis of variance (ANOVA) is performed. Otherwise, when the variance is not uniform, Brown-Forsythe and Welch tests are used. $P<0.05$ is considered statistically significant.

\section{Results}

The study subjects were images of 6 groups of different energies from 38 patients with non-small cell lung carcinoma. For the 228 plans obtained, CT values of tissues, target volume, target dose and organendangered dose were included as comparative indicators for analysis. All measurement data conform to the normal distribution. The results are expressed as the mean plus or minus standard deviation $(\bar{\chi} \pm S D)$, with two significant digits after the decimal point and three significant digits after the decimal point for the mean of $\mathrm{HI}$ and $\mathrm{Cl}$.

\subsection{Comparison of CT values of different energy CT images}

After transmitting the CT images to the planning system, the physician contoured the GTV, CTV, PTV and OAR(the left lung, right lung, heart, and spinal cord). Then, 5 points are randomly selected within each 
contoured structure, and the CT value of the corresponding points is read by the planning system, and the arithmetic average of the CT values of these 5 points is used as the CT value of the tissue. In this study, the CT values GTV, heart, lung, and spinal cord were selected for univariate analysis of variance. The results are shown in Table 1 :

Table 1

CT values obtained from different energy groups

\begin{tabular}{|lllll|}
\hline Group & GTV & Heart & Lung & Spinal Cord \\
\hline $40 \mathrm{keV}$ & $147.97 \pm 69.98$ & $318.63 \pm 54.28$ & $-782.42 \pm 65.17$ & $73.88 \pm 27.81$ \\
\hline $60 \mathrm{keV}$ & $78.38 \pm 33.11$ & $159.95 \pm 23.06$ & $-818.26 \pm 57.80$ & $45.18 \pm 13.60$ \\
\hline $80 \mathrm{keV}$ & $52.98 \pm 17.88$ & $97.29 \pm 12.55$ & $-831.63 \pm 55.08$ & $30.29 \pm 8.23$ \\
$100 \mathrm{keV}$ & $39.82 \pm 12.59$ & $67.66 \pm 12.23$ & $-838.08 \pm 54.12$ & $24.54 \pm 6.60$ \\
\hline $120 \mathrm{keV}$ & $35.01 \pm 9.31$ & $56.54 \pm 8.04$ & $-840.44 \pm 53.30$ & $22.38 \pm 5.50$ \\
\hline $140 \mathrm{keV}$ & $31.67 \pm 7.95$ & $48.86 \pm 7.79$ & $-842.19 \pm 53.19$ & $20.78 \pm 5.20$ \\
\hline$F$ value & $\mathrm{N} / \mathrm{A}$ & $\mathrm{N} / \mathrm{A}$ & $\mathrm{N} / \mathrm{A}$ & $\mathrm{N} / \mathrm{A}$ \\
\hline$P$ value & $\mathrm{N} / \mathrm{A}$ & $\mathrm{N} / \mathrm{A}$ & $\mathrm{N} / \mathrm{A}$ & $\mathrm{N} / \mathrm{A}$ \\
\hline $\begin{array}{l}\text { Brown-Forsythe* } \\
\text { W }\end{array}$ & 0.000 & 0.000 & 0.000 & 0.000 \\
\hline $\begin{array}{l}\text { Welch } \\
\text { N }\end{array}$ & 0.000 & 0.000 & 0.000 & 0.000 \\
\hline $\begin{array}{l}\text { Note: N/A illustrates that the variance is not uniform, this value is not applicable; Brown-Forsythe * is } \\
\text { the significant result of Brown-Forsythe test, Welch * is the significant result of Welch test, and when } \\
\text { the significant result is less than 0.05, the difference is statistically significant. }\end{array}$ & \\
\hline
\end{tabular}

As can be seen from the table above, the P values for the CT values of the four tissues are all less than 0.05 , and the differences in CT values of the same patient at different energy levels are statistically significant. Figure 1 shows the graphs of CT values for different tissues under different energies, which indicated that the CT values of the four tissues decreased with increasing energy. The range of CT values of the heart and lung varies widely, while that of the spinal cord is small.

\subsection{Comparison of differences in GTV contouring}

On the planning system, the volume of the GTV contoured by the physician was obtained. The results are shown in Table 2. Which shows that the $P$ value was greater than 0.05 , so the difference was not statistically significant. 
Table 2

The volume of the GTV for different

energies

\begin{tabular}{|lll|}
\hline Group & $\mathbf{n}$ & GTV Volume \\
\hline $40 \mathrm{keV}$ & 8 & $105.38 \pm 140.98$ \\
\hline $60 \mathrm{keV}$ & 8 & $102.51 \pm 132.75$ \\
\hline $80 \mathrm{keV}$ & 8 & $102.65 \pm 135.83$ \\
\hline $100 \mathrm{keV}$ & 8 & $100.91 \pm 132.12$ \\
\hline $120 \mathrm{keV}$ & 8 & $100.17 \pm 131.66$ \\
\hline $140 \mathrm{keV}$ & 8 & $101.64 \pm 135.99$ \\
\hline F value & & 0.001 \\
\hline$P$ value & & 1.000 \\
\hline
\end{tabular}

Six pairs of data above are compared pairwise. Among them, the $40 \mathrm{keV}$ energy group has the largest GTV volume, reaching $105.38 \pm 140.98 \mathrm{~cm} 3$, and the $120 \mathrm{keV}$ energy group has the smallest average volume. $\left(\mathrm{V}_{\max }-\mathrm{V}_{\min }\right) / \mathrm{V}_{\max } \times 100 \%$ was used to indicate the difference between the maximum and the minimum, and the value for GTV is $4.94 \%$. The smallest difference for GTV volume is between $60 \mathrm{keV}$ and $80 \mathrm{keV}$, which is only $0.14 \%$.

The CT images of different energies have different display details, as shown in Fig. 2.

\subsection{Dosimetric comparison of planning target areas}

All plans take PTV as the target area, and the PTV information including $D_{\text {max }}, D_{\text {mean }}, D_{\text {min }}, D_{2 \%}, D_{98 \%}$, $\mathrm{D}_{50 \%}, \mathrm{~V}_{\mathrm{PTV} 100 \%} \square \mathrm{V}_{\mathrm{PTV}}$, and $\mathrm{V}_{\mathrm{t}}$ of all 228 plans were obtained in order to get the $\mathrm{Cl}$ and $\mathrm{HI}$. And one-way analysis of variance was performed. The data are shown in Table 3.

From Table 3 we can see that, the maximum $\mathrm{HI}$ value is 0.166 , while the minimum value is 0.131 . When compared between groups, $P>0.05$, so the difference is not statistically significant. The maximum value of $\mathrm{Cl}$ is 0.721 , when the minimum value is 0.686 . Considering the comparison between groups, $\mathrm{P}>0.05$, obviously, the difference is not statistically significant. In summary, when planned with different energies, the uniformity and conformity of the target area are not affected by the energy. 
Table 3

Target dose analysis

\begin{tabular}{|llllll|}
\hline Groups & $\mathrm{HI}$ & $\mathrm{Cl}$ & $\mathrm{D}_{\max }$ & $\mathrm{D}_{\text {mean }}$ & $\mathrm{D}_{\text {min }}$ \\
\hline $40 \mathrm{keV}$ & $0.131 \pm 0.02$ & $0.719 \pm 0.06$ & $6775.31 \pm 100.36$ & $6272.15 \pm 45.29$ & $5050.05 \pm 681.04$ \\
\hline $60 \mathrm{keV}$ & $0.142 \pm 0.02$ & $0.709 \pm 0.06$ & $6798.03 \pm 165.11$ & $6301.31 \pm 63.79$ & $5033.33 \pm 655.13$ \\
\hline $80 \mathrm{keV}$ & $0.166 \pm 0.05$ & $0.721 \pm 0.06$ & $6917.22 \pm 302.17$ & $6364.31 \pm 111.46$ & $4940.53 \pm 684.68$ \\
\hline $100 \mathrm{keV}$ & $0.154 \pm 0.03$ & $0.686 \pm 0.07$ & $6858.11 \pm 209.30$ & $6335.30 \pm 72.32$ & $5104.70 \pm 430.31$ \\
\hline $120 \mathrm{keV}$ & $0.162 \pm 0.02$ & $0.704 \pm 0.08$ & $6929.90 \pm 159.26$ & $6367.44 \pm 80.49$ & $5124.29 \pm 391.38$ \\
\hline $140 \mathrm{keV}$ & $0.157 \pm 0.02$ & $0.687 \pm 0.07$ & $6864.74 \pm 195.86$ & $6345.21 \pm 72.15$ & $4988.88 \pm 654.46$ \\
\hline Fvalue & 1.879 & 0.400 & 0.780 & 1.896 & 0.108 \\
\hline Pvalue & 0.119 & 0.846 & 0.570 & 0.115 & 0.990 \\
\hline
\end{tabular}

When compared between groups, it can be shown from the table above that the $\mathrm{P}$ value was greater than 0.05 , and the maximum group of $D_{\text {max }}, D_{\text {mean }}$, and $D_{\text {min }}$ were all $120 \mathrm{keV}$ group, which were $6929.90 \pm$ $159.26,6367.44 \pm 80.49$, and $5142.29 \pm 391.38$ respectively. Both $D_{\max }$ and $D_{\text {mean }}$ minimum values appeared in the $40 \mathrm{keV}$ group, values $6775.31 \pm 100.36$ and $6772.15 \pm 45.29$, which meeting the requirements of the plan. The maximum and minimum values of $D_{\max }$ differ by $4.49 \%, D_{\text {mean }}$ by $1.49 \%$, and $D_{\min }$ by $3.58 \%$, all within $5 \%$.

\subsection{Dosimetric comparison of lung tissue, heart, and spinal cord}

\section{(1)Lung dose comparison}

The lungs were divided into affected lungs and healthy lungs for dose comparison. For both lungs, $V_{5}$, $V_{20}, V_{30}$ and $D_{\text {mean }}$ were evaluated respectively.

The lung is divided into the ipsilateral lung and the healthy lung for dose comparation, and the $V_{5}, V_{20}$, $V_{30}$ and $D_{\text {mean }}$ are evaluated. The results are shown in Tables 4 and 5. 
Table 4

Dose of the ipsilateral lung

\begin{tabular}{|c|c|c|c|c|c|}
\hline \multirow[t]{2}{*}{ Groups } & \multirow[t]{2}{*}{$\mathrm{n}$} & \multicolumn{4}{|l|}{ ipsilateral lung } \\
\hline & & V5 & V20 & V30 & $D_{\text {mean }}$ \\
\hline $40 \mathrm{keV}$ & 8 & $48.75 \pm 12.90$ & $31.03 \pm 7.11$ & $21.35 \pm 7.53$ & $1596.26 \pm 444.05$ \\
\hline $60 \mathrm{keV}$ & 8 & $52.36 \pm 14.22$ & $33.55 \pm 8.97$ & $24.12 \pm 9.62$ & $1690.15 \pm 483.26$ \\
\hline $80 \mathrm{keV}$ & 8 & $49.78 \pm 13.37$ & $30.78 \pm 6.93$ & $21.33 \pm 7.65$ & $1590.93 \pm 453.13$ \\
\hline $100 \mathrm{keV}$ & 8 & $50.00 \pm 13.09$ & $31.73 \pm 7.03$ & $21.88 \pm 7.26$ & $1618.33 \pm 434.29$ \\
\hline $120 \mathrm{keV}$ & 8 & $50.42 \pm 13.43$ & $31.61 \pm 7.39$ & $22.06 \pm 7.86$ & $1619.09 \pm 446.40$ \\
\hline $140 \mathrm{keV}$ & 8 & $50.19 \pm 13.28$ & $31.61 \pm 7.08$ & $21.86 \pm 7.52$ & $1616.73 \pm 427.58$ \\
\hline Fvalue & & 0.063 & 0.136 & 0.136 & 0.050 \\
\hline Pvalue & & 0.997 & 0.983 & 0.983 & 0.998 \\
\hline
\end{tabular}

Comparing the doses of 6 groups of the ipsilateral lung, $P>0.05$, which means the difference was not statistically significant. Among them, the doses of $\mathrm{V}_{5}, \mathrm{~V}_{20}, \mathrm{~V}_{30}$, and $\mathrm{D}_{\text {mean }}$ in the affected side of the $60 \mathrm{keV}$ group were the largest, and the differences from the minimum values were $6.89 \%, 8.26 \%, 11.57 \%$, and $5.87 \%$. The minimum doses of $\mathrm{V}_{20}, \mathrm{~V}_{30}$ and $\mathrm{D}_{\text {mean }}$ all appeared in the $80 \mathrm{keV}$ group, and the minimum dose of $\mathrm{V}_{5}$ was $40 \mathrm{keV}$.

Table 5

Dose of intact lung

\begin{tabular}{|c|c|c|c|c|c|}
\hline \multirow[t]{2}{*}{ Groups } & \multirow[t]{2}{*}{ n } & \multicolumn{4}{|l|}{ intact lung } \\
\hline & & V5 & V20 & V30 & Dmean \\
\hline $40 \mathrm{keV}$ & 8 & $28.69 \pm 17.93$ & $4.43 \pm 6.26$ & $0.51 \pm 0.73$ & $426.29 \pm 258.48$ \\
\hline $60 \mathrm{keV}$ & 8 & $30.81 \pm 16.46$ & $4.20 \pm 6.03$ & $0.43 \pm 0.60$ & $443.93 \pm 242.39$ \\
\hline $80 \mathrm{keV}$ & 8 & $29.94 \pm 17.58$ & $4.22 \pm 6.12$ & $0.47 \pm 0.65$ & $430.74 \pm 263.37$ \\
\hline $100 \mathrm{keV}$ & 8 & $30.59 \pm 17.62$ & $4.39 \pm 6.72$ & $0.39 \pm 0.57$ & $437.08 \pm 263.85$ \\
\hline $120 \mathrm{keV}$ & 8 & $30.78 \pm 18.20$ & $4.40 \pm 6.62$ & $0.48 \pm 0.67$ & $439.06 \pm 270.72$ \\
\hline $140 \mathrm{keV}$ & 8 & $30.67 \pm 17.68$ & $4.12 \pm 5.88$ & $0.45 \pm 0.60$ & $438.25 \pm 245.54$ \\
\hline Fvalue & & 0.018 & 0.003 & 0.036 & 0.005 \\
\hline Pvalue & & 1.000 & 1.000 & 0.999 & 1.000 \\
\hline
\end{tabular}


It can be seen from Table 5 that, similar to the ipsilateral lung, the dose data of the six groups of the healthy lung was compared, the result is $P>0.05$, so the difference was not statistically significant. Among them, the ipsilateral lungs in the $40 \mathrm{keV}$ group had the largest $V_{20}$ and $V_{30}$, while $V_{5}$ and $D_{\text {mean }}$ were the smallest. In the $60 \mathrm{keV}$ group, the ipsilateral lungs had the largest $V_{5}$ and $D_{\text {mean }}$. The minimum values of $V_{20}$ and $V_{30}$ appeared in the $140 \mathrm{keV}$ and $100 \mathrm{keV}$ groups, respectively. And the differences from the minimum values were $6.88 \%, 7.00 \%, 23.53 \%$ and $3.97 \%$. The dose curve of the ipsilateral and healthy lungs is shown in Fig. $3(a, b, c, d)$.

\section{(2) Comparison of cardiac and spinal doses}

Compare the $V_{30}, V_{40}, D_{\text {mean }}$ of the dose of the heart and the maximum dose $D_{\text {max }}$ of the spinal cord respectively. The data are shown in Table 6. It can be seen that, for the spinal dose of the six groups, $\mathrm{P}>$ 0.05 , for the $V_{30}, V_{40}$, and $D_{\text {mean }}$ of the heart dose, $P>0.05$. This shows that there is no statistically significant difference between the maximum dose of the spinal cord and that of the heart.

Table 6

Doses of heart and spinal cord

\begin{tabular}{|llllll|}
\hline Groups & $\mathbf{n}$ & Heart & & & Spinal cord \\
\cline { 3 - 6 } & & V30 & V40 & $\mathbf{D}_{\text {mean }}$ & $\mathrm{D}_{\text {max }}$ \\
\hline $40 \mathrm{keV}$ & 8 & $10.88 \pm 12.36$ & $4.38 \pm 5.47$ & $876.45 \pm 647.14$ & $3753.35 \pm 658.89$ \\
\hline $60 \mathrm{keV}$ & 8 & $7.24 \pm 10.28$ & $2.53 \pm 3.95$ & $833.71 \pm 638.26$ & $3739.28 \pm 656.60$ \\
\hline $80 \mathrm{keV}$ & 8 & $7.40 \pm 10.06$ & $2.50 \pm 3.89$ & $826.83 \pm 636.88$ & $3866.39 \pm 566.94$ \\
\hline $100 \mathrm{keV}$ & 8 & $8.13 \pm 10.03$ & $3.06 \pm 4.52$ & $857.55 \pm 638.26$ & $3941.00 \pm 823.45$ \\
\hline $120 \mathrm{keV}$ & 8 & $7.34 \pm 9.17$ & $2.55 \pm 3.46$ & $841.93 \pm 622.25$ & $3881.95 \pm 815.38$ \\
\hline $140 \mathrm{keV}$ & 8 & $7.96 \pm 10.06$ & $2.90 \pm 4.51$ & $850.33 \pm 642.91$ & $3807.34 \pm 680.48$ \\
\hline Fvalue & & 0.136 & 0.219 & 0.008 & 0.099 \\
\hline Pvalue & & 0.991 & 0.969 & 1.000 & 0.992 \\
\hline
\end{tabular}

From the Table 6 we can see that the maximum cardiac dose was in the $40 \mathrm{keV}$ group, in which the differences from the minimum were $33.46 \%, 42.92 \%$, and $5.66 \%$, respectively. The minimum value of $V_{30}$ was in the $60 \mathrm{keV}$ group, and the minimum value of $\mathrm{V}_{40}$ and $\mathrm{D}_{\text {mean }}$ was in the $80 \mathrm{keV}$ group. The maximum value of the spinal cord dose was $3941.00 \pm 823.45$, which appeared in the $100 \mathrm{keV}$ group, and the minimum value was $3739.28 \pm 656.60$, and appeared in the $60 \mathrm{keV}$ group. The difference between the maximum and minimum values was $5.12 \%$. Cardiac and spinal cord dose curves are shown in Fig. $3(e, f)$. 
In summary, there are slight differences in single-energy images of different energies obtained from spectral CT scans when they are used in the design of radiotherapy plans, which are expressed as: there are differences in the CT values of images obtained by different energies, but no significant impact on physicians target delineation. In terms of dose, when the radiotherapy plan is performed on CT images of different energies, the difference between $\mathrm{HI}$ and $\mathrm{Cl}$ values of the target area is not statistically significant. The differences between the various dose indicators are not significant. For the comparison between groups, the target area dose difference is the smallest (maximum 4.49\%). The difference between lung dose, heart dose and spinal dose is in $3.97 \%-42.92 \%$, obviously, the difference is large, but these differences are not statistically significant.

\subsection{Discussion}

The interactions between X-rays and substances mainly include photoelectric effect, Compton effect and electron pair effect. The commonly used diagnostic X-ray energy in the clinic is between $30 \sim 200 \mathrm{keV}$ [16]. In this energy band, the photoelectric effect is dominant, and the mode of interaction depends on the characteristics of the substance itself and the X-ray energy. The lower the energy, the stronger the ability to distinguish substances [17]. Based on this characteristic, the traditional CT imaging method is attenuation imaging. The current CT image reconstruction algorithms include: filtered back projection algorithm, iterative reconstruction algorithm and direct Fourier back projection algorithm. Dual-energy computed tomography (DECT) reconstruction methods mainly include pre-processing method, postprocessing method and iterative method [18]. Image reconstruction method is an influencing factor of image error.

Jiang Jie et al. [19] studied the diagnosis of chest diseases using dual-source CT virtual plain scan technology. Their results showed that the image quality of the virtual plain scan was not statistically different from that of the conventional plain scan. But for the display of partial calcifications and small lymph nodes near the vena cava, the plain scan group is superior to the virtual plain scan. Yan Zhaoxian et al.[20] studied the image quality of head and neck dual-energy low-dose radiation and found that compared with conventional doses, the signal-to-noise ratio (SNR) and image quality scores of the lowdose group images were not statistically significant. Zhang Xintang et al. [21] compared the enhanced images of lung cancer obtained by dual-energy scanning and conventional scanning, and found that the images obtained by the two scanning methods have no difference in the detection rate of the lesion and the detailed display of lobulation sign, burr sign, cavity, necrosis and so on. The studies above compared the difference between ordinary scanning and energy spectrum scanning imaging. Our research refines the difference in target area delineation caused by different reconstruction energy of energy spectrum scanning. The results show that the GTV volume difference obtained from the images of 6 groups of energy is not statistically significant.

Li Mingying et al. [22] believed that the lower the X-ray energy, the greater the absorption coefficient. The more X-ray attenuation, the better the CT value at $40 \mathrm{keV}$ reflects the nature of the substance. The CT value of tumor tissue of lung adenocarcinoma and squamous cell carcinoma decreases with the increase of energy. This trend is consistent with the results of this study. Yan Zhaoxian et al. [20] believed that 
there was no difference in the CT values of blood vessels obtained by different doses of CT imaging. Mitchell et al. [23] used phantom scanning and theoretical calculations to find that as the energy changes between 40 and $120 \mathrm{keV}$, the CT root-mean-square error varies between $6 \mathrm{HU}$ and $248 \mathrm{HU}$, Especially for the combination of low energy and high atomic number, the difference is more obvious. In this study, the maximum CT value difference was within the GTV of the $40 \mathrm{keV}$ group, reaching $231 \mathrm{HU}$. Qi Zhenyu et al [24] showed that the scanning voltage, CT bed surface and geometric position of the phantom are all influencing factors of CT value. In this study, the CT values of GTV, heart and spinal cord tissues all decreased with increasing energy. The significant difference in CT value did not have a significant impact on the volume of the GTV outlined by the physician. The author believes that this may be related to the recognition ability of the human eyes. Under the condition of a specific window width and window level, if the gray difference between adjacent areas is less than IMax / 16, it is difficult for the human eye to distinguish [25]. With the development of artificial intelligence, automatic contouring technology has received extensive attention [26]. The contouring technology based on CT values will inevitably place higher demands on the accuracy of the CT values of the images.

The dose calculation of the radiotherapy planning system depends on the CT value-electron density curve. The CT value obtained from the phantom scan under specific scanning conditions and the electronic density value provided by the manufacturer are input during data configuration. Gao Liquan et al [27] used different CT-electron density conversion curve, the difference of MU values obtained by different plans are all $<20 \%$, of which the intensity modulation plan is larger. In recent years, scholars have done a lot of research on the relationship between CT value and electron density curve. A lot of research has been done [28-32]. Qin Jiaojiao et al proposed a spectral CT electron density image reconstruction technology based on multi-material decomposition algorithm. Numerical simulation experiment results It is shown that the electron density image reconstructed by this algorithm can easily and intuitively distinguish four substances: water, ethanol, glycerol and plexiglass, and the internal structure information of the image is more abundant. This indicates that the spectral CT is used for both diagnosis and radiation therapy. There is huge potential.

\section{Conclusions}

In this study, under different reconstruction energies, the CT value of the same tissue is different, which in turn causes the difference in radiotherapy dose. Since our study uses the same CT value-electron density curve, the accuracy of the calculation of each energy dose still needs to be further improved. In the following work, future research in this area can be refined in order to provide a reference for the selection of scanning conditions for precision radiotherapy.

\section{Abbreviations}

CT

Computer Tomography

OARs 
organs at risk

PTV

planning target volume

GTV

gross tumor volume

$\mathrm{HI}$

homogeneity index

$\mathrm{Cl}$

conformity index

NSCLC

Non-small cell Lung Cancer

CTV

clinical target volume

IMRT

intensity modulated radiation therapy

DECT

Dual-energy computed tomography

SNR

signal-to-noise ratio

\section{Declarations}

\section{Ethical Approval and Consent to participate}

This study was approved by the institution Ethics Commission of The First Affiffiffiliated Hospital of Shandong First Medical University. The need for consent to participate was waived by Ethics Commission of The First Affiffiffiliated Hospital of Shandong First Medical University.

\section{Consent for publication}

Written informed consent was obtained from each patient.

\section{Availability of supporting data}

Please contact authors for data requests.

\section{Competing interests}

The authors declare that they have no competing interests.

\section{Funding}


Yong Hou received Projects of medical and health technology development program in Shandong province(2017WS790)

\section{Authors' contributions}

Yong Hou made substantial contributions to the conception design of the work. Tian Xiufang was a major contributor in writing the manuscript. Liu Kun and Zhang Jian Dong substantively revised the manuscipt. Wang Jing analyzed the data and created figures using data. All authors read and approved the final manuscript.

\section{Acknowledgements}

Not applicable

\section{References}

[1] Hounsfield, G. N. Computerized transverse axial scanning (tomography): Part 1. Description of system[J]. The British Journal of Radiology, 1973, 46(552):1016-1022. PubMed

[2] Wang Qi, Chen Zhiqiang, Wu Xiaoping, et al. Overview of X-ray safety inspection technology [J]. CT Theory and Application Research, 2004,13 (1): 32-37. WanFang

[3] Han Xinxin, Zhang Yun, Liu Wei, et al. Application of dual-energy CT in diagnosis and treatment of gout [J] .Chinese Journal of Clinical Immunology and Allergy, 2017,11 (04): 370-374. WanFang

[4] Primak A N , Fletcher J G , Vrtiska T J , et al. Noninvasive Differentiation of Uric Acid versus Non-Uric Acid Kidney Stones Using Dual-Energy CT[J]. Academic Radiology, 2007, 14(12):1441-1447. PubMed

[5] Foti Giovanni, Catania Matteo, Caia Simone, etal. Identification of bone marrow edema of the ankle: diagnostic accuracy of dual-energy CT in comparison with MRI.[J]. La Radiologia medica,2019,124(10). PubMed

[6] Lin Xiaozhu, Li Weixia, Zhu Yanbo, et al. Preliminary Application of Gem Energy Spectrum CT in Tumor Diagnosis [J]. Journal of Diagnostics Theory and Practice, 2010,9 (2): 155-160. WanFang

[7] Tian Shifeng, Liu Ailian, Wang Heqing, et al. Diagnostic value of single-source dual-energy CT iodine base map for early gastric cancer [J] .Chinese Journal of Clinicians (Electronic Edition), 2016,10 (23): 3674-3677. Baidu Scholar

[8] Chae E J , Song J W , Seo J B , et al. Clinical Utility of Dual-Energy CT in the Evaluation of Solitary Pulmonary Nodules: Initial Experience[J]. Radiology, 2008, 249(2):671-681. PubMed

[9] Chae E J , Song J W , Krauss B , et al. Dual-energy Computed Tomography Characterization of Solitary Pulmonary Nodules[J]. Journal of Thoracic Imaging, 2010, 25(4):301-310. PubMed 
[10] Ren Qingguo, Hua Yanqing, Li Jianying.The basic principle and clinical application of CT energy spectrum imaging [J] .International Journal of Medical Radiology, 2011,34 (06): 559-563. WanFang

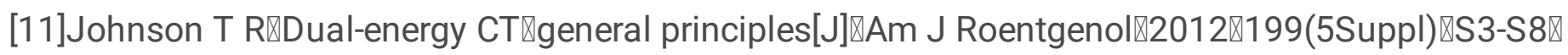
PubMed

[12] KRY S F, BEDNARZ B, HOWELL R M, et al. AAPM TG158: measurement and calculation of doses outside the treated volume from external-beam radiation therapy $\mathbb{J} \mathbb{\text { }}$. Med Phys, 2017, 44(10): e391e429. PubMed

[13] Dai Xiangkun, Wang Yunlai, Feng Linchun, et al. Research on MVCT images of spiral tomography accelerator used for head and neck dose calculation [J] .Chinese Journal of Medical Devices, 2014,38 (02): 141-144. WanFang

[14] Zhou Dingyi, Jiang Dazhen, Quan Hong, et al. The influence of four-dimensional CT scanning parameters and scanning modes on CT values [J] .Chinese Journal of Medical Physics, 2016,33 (09): 919-923. WanFang

[15] V. Grégoire, Mackie T R . State of the art on dose prescription, reporting and recording in IntensityModulated Radiation Therapy (ICRU report No. 83)[J]. Cancer/radiothérapie, 2011, 15(6-7):0-559. PubMed

[16] Tsunoo T , Torikoshi M , Sasaki M , et al. Distribution of Electron Density Using Dual-Energy X-Ray CT[J]. IEEE Transactions on Nuclear Science, 2003, 50(5):1678-1682. Baidu Scholar

[17]Hu Yimin. Radiation Physics [M]. Beijing: Atomic Energy Press, 1999. WanFang

[18] Zheng Peng, Hao Jia, Xing Yuxiang. Theoretical error analysis of dual-energy CT imaging base material decomposition method [J]. CT Theory and Application Research, 2011 (02): 3-12. WanFang

[19] Jiang Jie, He Bo, Zhang Jun, et al. Application value of dual-source CT dual-energy chest virtual flat scan [J]. Chinese Medical Imaging Technology, 2012 (04): 185-189. WanFang

[20] Yan Zhaoxian. Research on dual-energy CTA low radiation dose imaging of head and neck [D]. Guangzhou University of Chinese Medicine, 2015. WanFang

[21] Zhang Xintang, Shen Jin, Han Dan. The clinical value of dual-source CT dual-energy technology in the diagnosis of lung cancer [J]. Journal of Practical Radiology, 2013,29 (8): 1245-1248,1257. WanFang

[22] Li Mingying, Wang Guangli, Cui Dingye, et al. Preliminary study on classification of lung cancer by energy spectrum CT imaging [J]. Journal of Shandong University (Medical Edition), 2012, 50 (12): 7376. WanFang

[23] Goodsitt M M , Christodoulou E G , Larson S C . Accuracies of the synthesized monochromatic CT numbers and effective atomic numbers obtained with a rapid $k V p$ switching dual energy CT scanner[J]. 
[24] Qi Zhenyu, Huang Shaomin, Deng Xiaowu. Calibration detection of CT value of radiotherapy plan and analysis of its influencing factors [J]. Cancer, 2006 (01): 110-114. PubMed

[25] Luo Shuqian, Zhou Guohong. Medical image processing and analysis [M]. Beijing: Science Press, 2010. WanFang

[26] Qin Wenjian. Research on key issues of medical image segmentation based on machine learning and its application in tumor diagnosis and treatment [D]. University of Chinese Academy of Sciences (Shenzhen Advanced Technology Research Institute of Chinese Academy of Sciences), 2019. WanFang

[27] Gao Liquan, Sun Xiaozhe, Liu Zhihui. Acquisition and correction of CT-electron density conversion curve and its effect on dose calculation of treatment plan [J]. Medical and Health Equipment, 2016, 37 (02): 103-105. WanFang

[28] Qin Jiaojiao. Research on the application of dual-energy CT (DECT) electron density image reconstruction technology [D]. Shandong University, 2018. WanFang

[29] Saito M . Potential of dual-energy subtraction for converting CT numbers to electron density based on a single linear relationship[J]. Medical Physics, 2012, 39(4):2021-2030. PubMed

[30] Hünemohr, Nora, Krauss B, et al. Experimental verification of ion stopping power prediction from dual energy CT data in tissue surrogates[J]. Physics in Medicine \& Biology, 2014, 59(1):83-96. PubMed

[31] Bourque A E , Carrier J F , Bouchard H. A stoichiometric calibration method for dual energy computed tomography[J]. Physics in Medicine and Biology, 2014, 59(8):2059-2088. PubMed

[32] van Abbema Joanne K, van Goethem Marc-Jan, Greuter Marcel J W, etal. Relative electron density determination using a physics based parameterization of photon interactions in medical DECT.[J]. Physics in medicine and biology,2015,60(9):3825-3846. PubMed

\section{Figures}




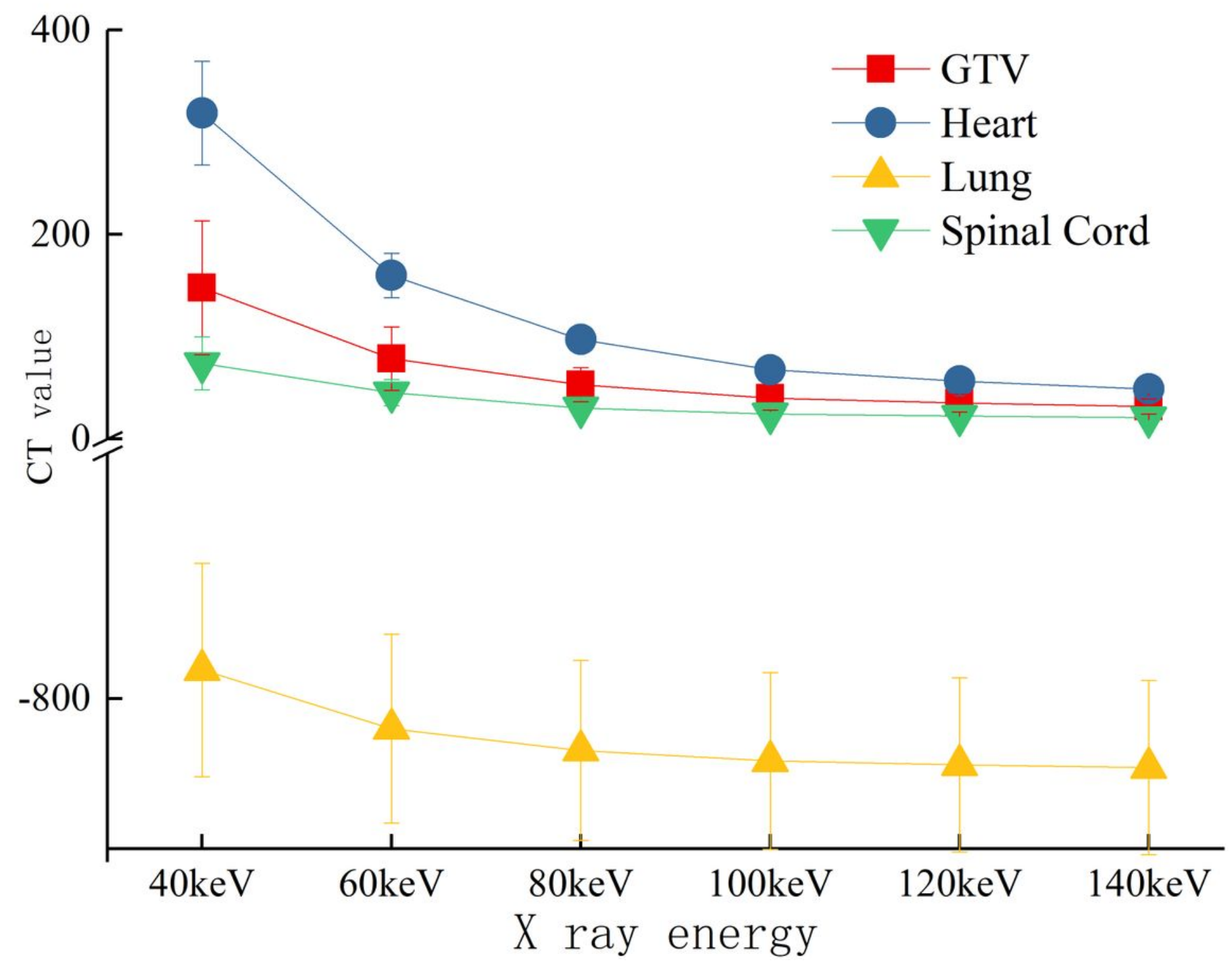

Figure 1

Graphs of CT values for different tissues under different energies 


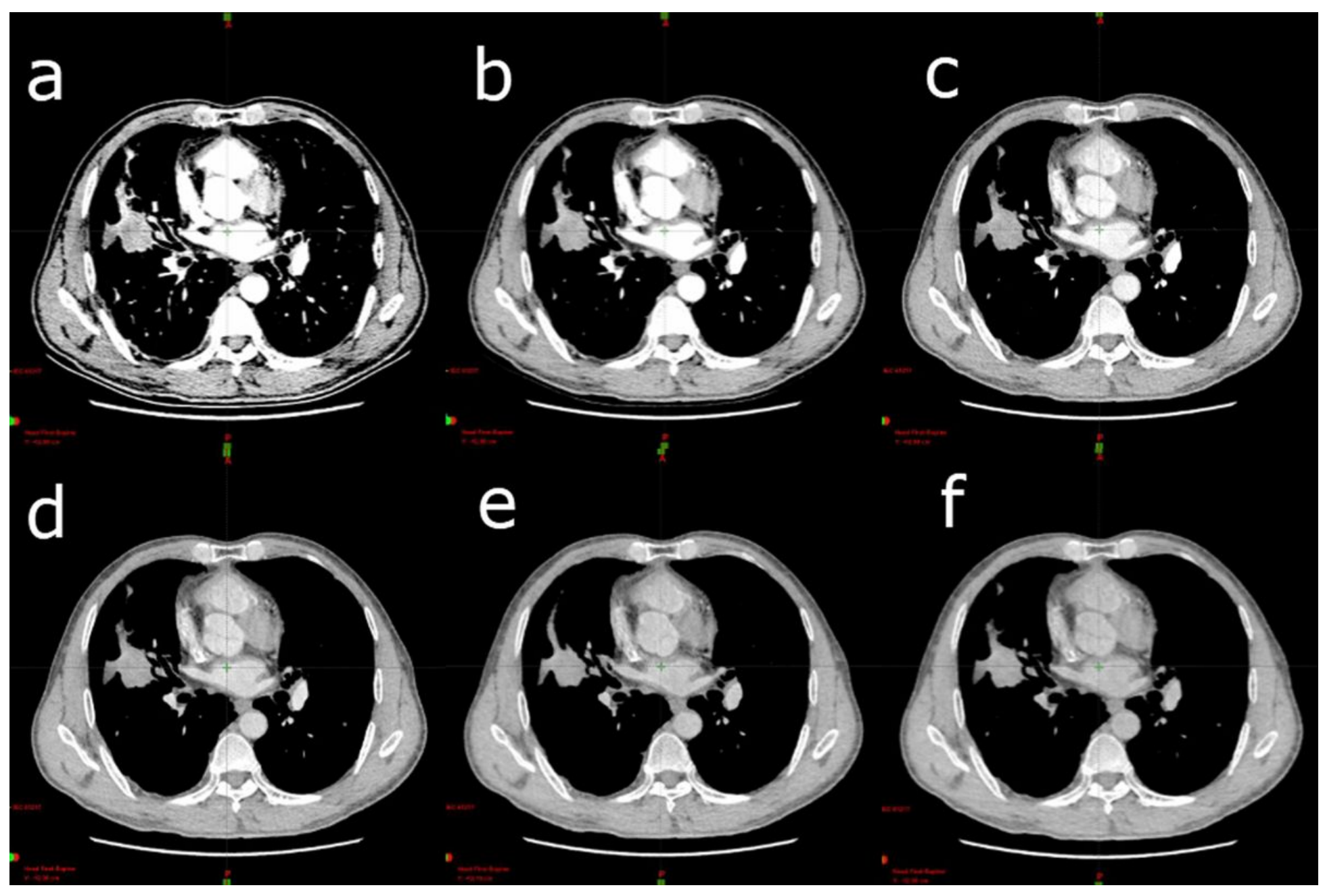

Figure 2

CT images of different energies on the same level (W / L: 400/40) Note: The energy represented by serial numbers $1-6$ are $40 \mathrm{keV}, 60 \mathrm{keV}, 80 \mathrm{keV}, 100 \mathrm{keV}, 120 \mathrm{keV}, 140 \mathrm{keV}$ 


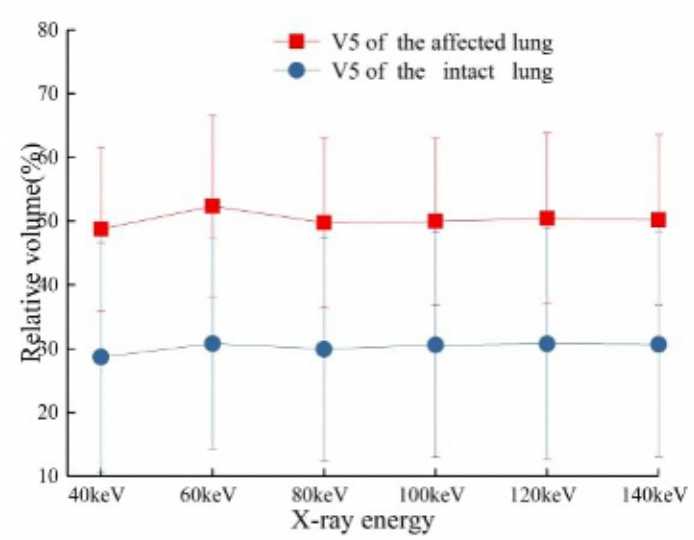

Figure3(a) V5 of the lungs

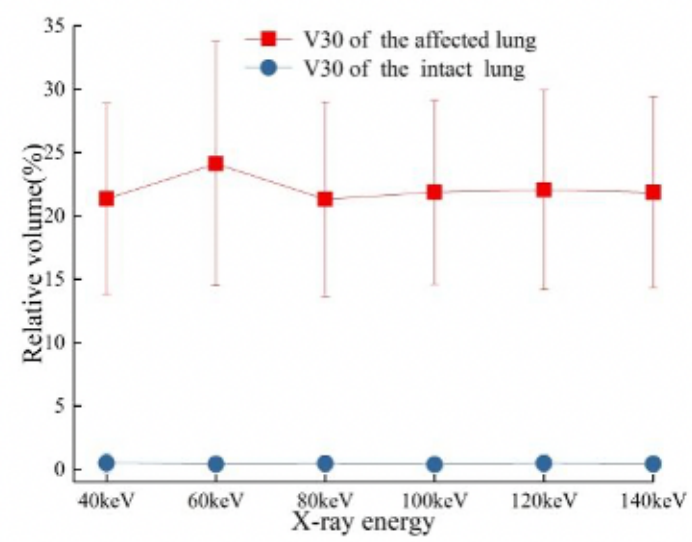

Figure3(c) V30 of the lungs

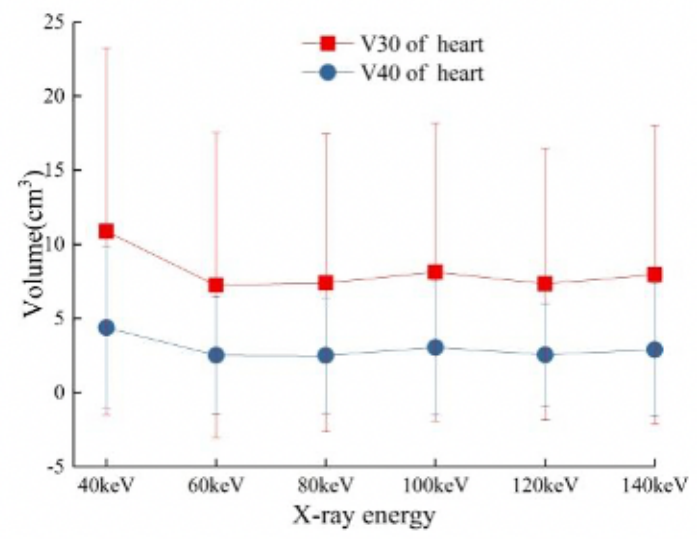

Figure 3(e) V30 and V40of the heart dose

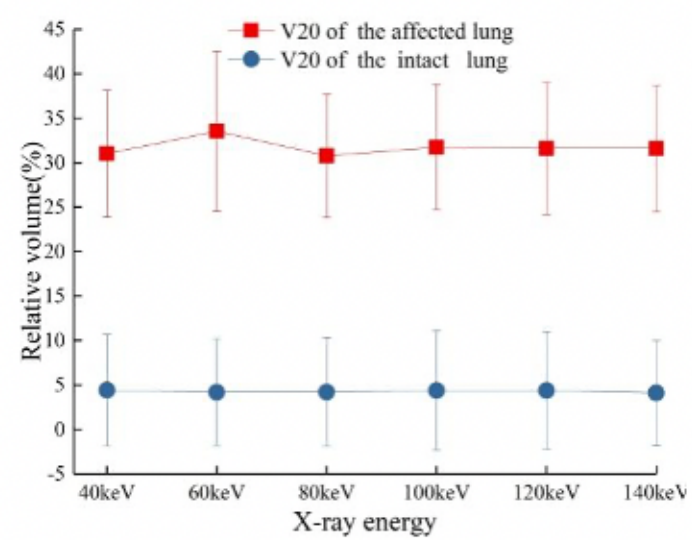

Figure3(b) V20 of the lungs

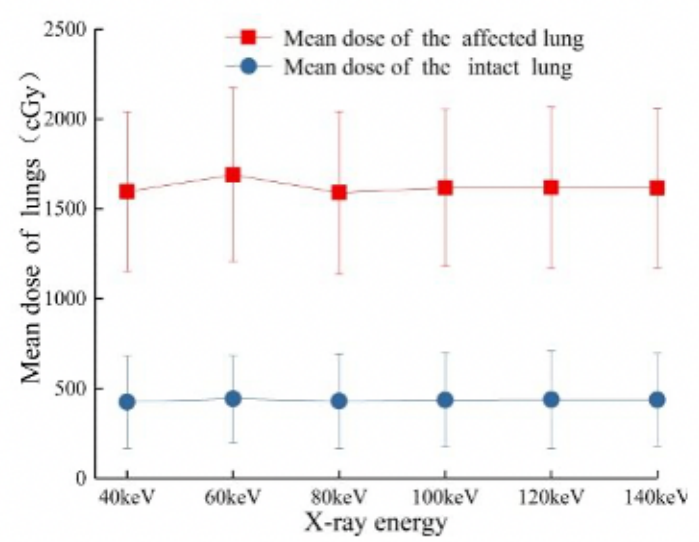

Figure3(d) Mean dose of the lungs

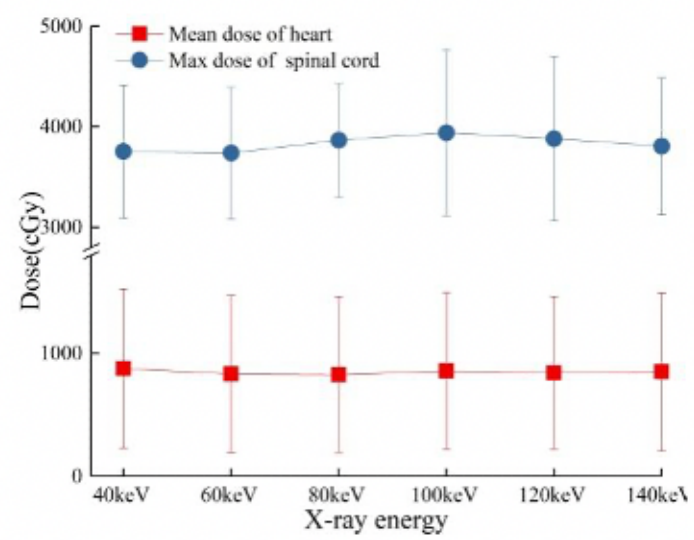

Figure 3 (f) Mean dose of the heart and maximum dose of the spinal cord

\section{Figure 3}

Dose of organ at risk 